\title{
Borderline personality disorder in adolescents: prevalence, diagnosis, and treatment strategies
}

This article was published in the following Dove Press journal:

Adolescent Health, Medicine and Therapeutics

\author{
Jean Marc Guilél,2,3 \\ Laure Boissel ${ }^{1,2}$ \\ Stéphanie Alaux-Cantin ${ }^{1,2}$ \\ Sébastien Garny de La \\ Rivière' \\ 'Child and Adolescent Psychiatry \\ Services, Amiens-Picardie University \\ Medical Centre, Amiens, France; \\ ${ }^{2}$ Psychiatry Residency Program, \\ University of Picardie-Jules Verne, \\ Amiens, France; ${ }^{3}$ Department \\ of Psychiatry, McGill University, \\ Montreal, Quebec, Canada
}

\begin{abstract}
Using the same Diagnostic and Statistical Manual of Mental Disorders, fifth version $(D S M-V)$ criteria as in adults, borderline personality disorder (BPD) in adolescents is defined as a 1-year pattern of immature personality development with disturbances in at least five of the following domains: efforts to avoid abandonment, unstable interpersonal relationships, identity disturbance, impulsivity, suicidal and self-mutilating behaviors, affective instability, chronic feelings of emptiness, inappropriate intense anger, and stress-related paranoid ideation. BPD can be reliably diagnosed in adolescents as young as 11 years. The available epidemiological studies suggest that the prevalence of BPD in the general population of adolescents is around $3 \%$. The clinical prevalence of BPD ranges from $11 \%$ in adolescents consulting at an outpatient clinic to $78 \%$ in suicidal adolescents attending an emergency department. The diagnostic procedure is based on a clinical assessment with respect to developmental milestones and the interpersonal context. The key diagnostic criterion is the 1-year duration of symptoms. Standardized, clinician-rated instruments are available for guiding this assessment (eg, the Diagnostic Interview for Borderlines-Revised and the Childhood Interview for DSM-IV-TR BPD). The assessment should include an evaluation of the suicidal risk. Differential diagnosis is a particular challenge, given the high frequency of mixed presentations and comorbidities. With respect to clinical and epidemiological studies, externalizing disorders in childhood constitute a risk factor for developing BPD in early adolescence, whereas adolescent depressive disorders are predictive of BPD in adulthood. The treatment of adolescents with BPD requires commitment from the parents, a cohesive medical team, and a coherent treatment schedule. With regard to evidence-based medicine, psychopharmacological treatment is not recommended and, if ultimately required, should be limited to second-generation antipsychotics. Supportive psychotherapy is the most commonly available first-line treatment. Randomized controlled trials have provided evidence in favor of the use of specific, manualized psychotherapies (dialectic-behavioral therapy, cognitive analytic therapy, and mentalization-based therapy).
\end{abstract}

Keywords: borderline personality, adolescence, prevalence, assessment, treatment

\section{Introduction}

The clinical existence of borderline personality disorder (BPD) in adolescence has long been debated. However, the disorder has now been better ascertained, and the evidence justifies the diagnosis and management of BPD in adolescents. ${ }^{1-3}$ BPD comprises an interiorized component (identity disturbance, stress-related paranoid ideation, chronic feelings of emptiness, and efforts to avoid abandonment), an emotional component (affective instability and inappropriate, intense anger), and an externalized component
Correspondence: Jean Marc Guilé Child and Adolescent Psychiatry Services, Amiens-Picardie University Medical Centre, Avenue Laënnec - 80480, Amiens, France

Email guile.jean-marc@chu-amiens.fr 
(impulsivity, suicidal and self-mutilating behaviors, and interpersonal relationships instability $)^{4}$ (Table 1). In fact, the affective instability corresponds to hypervigilance and affective hyperreactivity. It manifests itself as a markedly unstable emotional state that depends on the subject's environment, interactions, and the responses elicited by the subject's requests and behaviors. Affective instability may be the key factor in adolescents treated as outpatients, ${ }^{4}$ whereas the identity disturbance may be the most important factor in hospitalized adolescents, ie, those suffering from the most severe forms of BPD. ${ }^{5}$ The clinical presentation as a whole is marked by instability. The intensity and duration of the symptoms fluctuate as a function of the affective context and the degree to which the situation is experienced as stressful by the adolescent.

As described notably in the Diagnostic and Statistical Manual of Mental Disorders, fifth version (DSM-V), ${ }^{6}$ the concept of BPD translates the broader psychoanalytic concept of a borderline personality organization (BPO, referred to as an "état limite" (borderline state) by French-speaking authors) into observable, semiological criteria. Hence, BPD is one of a broader variety of personality disorders through which a BPO can be symptomatically expressed. ${ }^{1,7}$ Historically, the BPD construct was derived from psychoanalytical work; however, it has been examined from neuroscientific and genetic standpoints for more than a decade now. ${ }^{8}$

Several studies have screened adolescents for the cognitive and neuronal correlates of BPD observed in adults. With regard to information processing, the tendency to negatively interpret neutral or ambiguous facial emotions in others is not observed in the adolescent; this might only be a feature of the most severe forms or might arise later in the development of BPD. In neuronal terms, hyperactivity of the amygdala and hippocampus has been observed not only in adolescent females with repeated self-injury and depression ${ }^{9}$

Table I Shortened list of criteria for borderline personality disorder, according to DSM-5

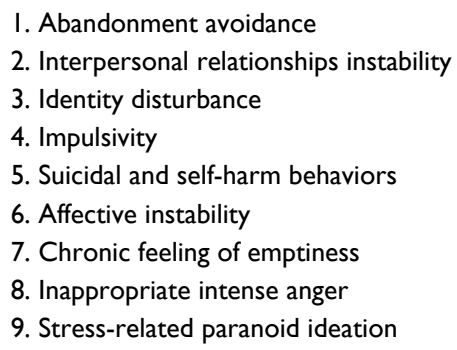

Source: APA, 2013.6

Abbreviation: DSM-5, Diagnostic and Statistical Manual of Mental Disorders, fifth version. but also in nondepressed adolescent females with BPD. ${ }^{10}$ Exposure to environmental stressors modifies the activation of these amygdala networks. With regard to hyperactivity of the amygdala, research has highlighted patterns of reactiveness to stressors, with hyper-reactivity of the corticotropic axis and then the development of hypo-reactivity (when the exposure to stressors becomes chronic) or the maintenance of hyper-reactivity when BPD is combined with depression. ${ }^{11}$ The activity of these neuronal networks (and notably that of the amygdala networks) is determined not only by environmental stressors but also by genetic vulnerability factors. Several candidate genetic factors have been explored, including the serotonin-transporter-linked polymorphic region (5-HTTLPR) and the $N R 3 C 1$ gene encoding the glucocorticoid receptor. ${ }^{12}$ Adversity in early life (maltreatment, in particular) modifies the neuronal and cognitive processes underlying the stress response via epigenetic mechanisms. ${ }^{13}$

The relationships between these mechanisms and vulnerability factors (both genetic and environmental) must be considered from a developmental perspective. These relationships are bidirectional and fluctuating, since they strengthen or weaken as the adolescent interacts with his/her family and peers. ${ }^{14}$ Early adversity, maltreatment, and maternal separation are associated with BPD., ${ }^{2,15}$ These early risk factors disrupt attachment, which in turn produces psychopathologic conditions such as BPD. ${ }^{16}$ In a family environment marked by early relationship instability, maltreatment, the absence of emotion-containing parenting, ${ }^{17}$ and disorganized attachment, the youngster's progression depends on his/her ability to build a coherent self-image. In fact, the persistence of a disorganized attachment style prevents the development of mentalization abilities. ${ }^{18}$ The child cannot pull together the succession of emotions experienced with his/her family and friends into a single, coherent image. Parental figures do not constitute internal objects (in the psychodynamic sense) that contribute to the youngster's psychological development.

On the psychological level, a BPO, as defined within a psychodynamically oriented perspective, results from the failure to interiorize and identify with reassuring, emotioncontaining, and structure-giving objects - leaving the adolescent confronted with nonsense, emptiness, and his/ her internal destructiveness. ${ }^{17}$ Psychological functioning is organized around a permanently unstable relationship with objects (rather than internal conflict), repeating the early confrontation with absent, unpredictable, or maltreating external objects. This translates into identity disturbance (a $D S M-V$ criterion). The adolescent feels very different and lacks coherence at certain times and in certain life situations. 
When interacting with others, the adolescent does not adequately perceive the boundary between his/her own ideas and emotions and those of other people. These observations from the field of clinical psychodynamics have recently been operationalized (in adult BPD) into research studies of metacognitive capacities. ${ }^{19}$ To the best of our knowledge, this type of study has not yet been performed in the adolescent.

In summary, research evidence from the biological, psychological, and social domains supports a stress-diathesis model of BPD. ${ }^{2}$ In turn, understanding an adolescent's clinical situation with regard to this model results in a more appropriate management and treatment plan for BPD.

\section{Prevalence}

A number of large epidemiological studies (based on national registries) have documented the current increase in reports of BPD - including those in adolescents. A Danish study ${ }^{20}$ found that the incidence of BPD in females aged 15 years or over increased linearly from 1970 to 2009. A Canadian study ${ }^{21}$ of diagnostic information collected through the public health insurance system found a steady increase in the incidence of BPD in 14- to 17-year-old girls between 2000 and 2012. These studies captured data on individuals having received care in the public hospital system but excluded the least impaired cases (eg, those having received care in the private sector) and the most severely impaired cases (eg, those who ended up in detention without receiving care).$^{22}$ It is important to note that these studies highlight the diminishing reluctance of clinicians to diagnose BPD in adolescents, since the work was based on diagnostic data recorded by the adolescent's treating physicians.

Prevalence studies have been also conducted in various clinical populations, regardless of the institutions' public or private sector status. Although the clinical samples are heterogeneous, one observes an increase in the prevalence of BPD with the severity of the clinical picture and the type of psychiatric care received: $11 \%$ in outpatient adolescents, ${ }^{23}$ between $19 \%$ and $53 \%$ in hospitalized adolescents, ${ }^{24-26} 62 \%$ in hospitalized suicidal adolescents, ${ }^{27}$ and $78 \%$ in adolescents attending the emergency department for suicidal behaviors. ${ }^{28,29}$

There are few studies of BPD in the general population, ${ }^{30}$ and even fewer in adolescents. ${ }^{2,3}$ The various epidemiological studies of the general population differ significantly with regard to the number of DSM criteria required, the catchment area, and the composition of the study sample. The first study of a randomly selected sample of 733 American youngsters aged 9-19 years applied two severity thresholds, depending on the number of symptoms present. ${ }^{31}$ For severe BPD, the study found prevalences of $2.8 \%$ in boys and $3.8 \%$ in girls aged 11-14 years. For moderate BPD, the values were $8.3 \%$ in boys and $11.5 \%$ in girls. A later study ${ }^{32}$ of a representative sample in the same catchment area observed a markedly lower prevalence $(0.9 \%)$ at 14 and 16 years of age. Several estimate thresholds were tested in a Canadian study ${ }^{33}$ of a representative community-based sample of 799 adolescents aged between 12 and 14 years. ${ }^{33}$ Along with the DSM criteria, a criterion for impairment on the Columbia Impairment Scale (CIS) was added, providing several different severity thresholds. ${ }^{34}$ The prevalence rates for the sample as a whole ranged from $6.3 \%$ without the additional CIS criterion to $3.2 \%$ with the highest CIS threshold. When considering the most impaired adolescents within the whole sample, the prevalence was $2.6 \%$ for males and $3.9 \%$ for females. Within the most severely impaired adolescents, the prevalence was $1.3 \%$ for the youngest group (12 years of age). A French study using a threshold of 8 in the Diagnostic Interview for Borderlines-Revised (DIB-R) and a random sample of 14-year-olds reported a prevalence of $11 \%$ in boys and $26 \%$ in girls. ${ }^{35}$ As discussed later, this strikingly high prevalence could be partly explained by the absence of additional impairment criterion. The most recent study (performed in the UK) found much the same values as Bernstein et $\mathrm{al}^{31}$ in a cohort of 6,330 11-year-olds ( $2.8 \%$ in boys, $3.6 \%$ in girls, and $3.2 \%$ for the study sample as a whole). ${ }^{36}$ Last, a study of adolescents in Hong Kong reported a prevalence of $2 \% .{ }^{37}$

The first conclusion that can be drawn from the results of these population studies is that the prevalence values differ markedly from one study to another (eg, values of between $0.9 \%{ }^{32}$ and $26 \%{ }^{35}$ in adolescents aged 14 years) or when comparing studies performed in the same catchment area (from $0.9 \%^{32}$ to a range of $2.8 \%-11.5 \%{ }^{31}$ ). The epidemiological objective, the diagnostic instruments used (which were nevertheless relatively homogeneous), and, above all, the algorithm used to determine the severity thresholds and the prevalence values contribute to these disparities. For example, Johnson et $\mathrm{a}^{32}$ investigated the cumulative prevalence of BPD rather than the prevalence rates for each age range. Consequently, the algorithm computed not only the youth's response at the age of 14 years but also the response assigned to the same individual 2 years later. Therefore, the value of $0.9 \%$ corresponded to individuals who retained a diagnosis of $\mathrm{BPD}$ throughout the assessments (ie, between 14 and 16 years of age). The estimation of prevalence depends not only on the number of diagnostic criteria fulfilled but also on whether additional criteria are added. For example, adding a severity 
criterion strengthens an estimate's validity. As in Bernstein et al's original study, ${ }^{31}$ many studies have used an intrinsic methodology to determine this threshold, eg, the number of diagnostic criteria present in addition to the number required to establish a diagnosis. ${ }^{38}$ However, the use of this type of intrinsic methodology means that the measurement of severity is not independent of the diagnostic measurement. ${ }^{39}$ On the contrary, the Canadian study ${ }^{33}$ used thresholds based on an impairment scale; this enabled independent measurements and fulfilled the $D S M-V$ 's criterion $\mathrm{C}$ for personality disorders and criterion $\mathrm{G}$ of the alternative DSM- 5 model ${ }^{6}$ On the same lines, the absence of an additional impairment criterion may have contributed to the high prevalence rate $(26 \%)$ observed in the school sample studied by Chabrol et al. ${ }^{35}$

Given that not all the studies systematically presented their estimations of prevalence by age and by gender, it is not always easy to determine the relative weights of these two factors in the observed interstudy differences. In the Canadian study, ${ }^{33}$ the prevalence rates increased with age; for the most severe cases, the prevalence rate ranged from $1.3 \%$ in the youngest (12-year-old) group to $4.5 \%$ for the oldest (14-year-old) group. Taken together, adolescents as young as 11 or 12 years were able to describe behaviors pertaining to BPD with enough accuracy for a diagnosis of BPD to be considered.

This is consistent with clinical observations. ${ }^{1}$ Although BPD has not been recognized in childhood, the clinical relevance of this diagnosis in adolescence is now well established. In keeping with these results, a sizeable proportion of adolescents (including some as young as 11 or 12) may continue to experience BPD in adulthood, with its associated negative outcomes and a high likelihood of not receiving timely, appropriate psychiatric care. Considering the prevalence rates observed among 11- and 12-year-old adolescents in the British and Canadian studies, respectively, prevention-focused interventions should target professionals dealing with adolescents (especially youth/social workers and school health staff) and provide them with information on borderline personality traits (BPTs) in early adolescence and how to identify them.

In summary, community-based studies have demonstrated that BPD can be identified in adolescents as young as 11 years..$^{31,36}$ With regard to the available literature, ${ }^{31,33,36}$ a conservative prevalence estimate would be around $3 \%$ in the general population of adolescents. Clinical studies have yielded much higher and more variable prevalence rates, starting at $11 \%$ in organic personality disorder (OPD) samples ${ }^{23}$ and peaking at $78 \%$ in suicidal adolescents referred to the emergency department. ${ }^{29}$ Not all prevalence studies consistently reported on sex characteristics. Significantly higher prevalence for girls has been observed in one clinical study $^{40}$ and three epidemiological studies. ${ }^{33,41,42}$

\section{Diagnosis}

On consultation, many adolescents (particularly those in an emergency or crisis situation) present with BPTs. When faced with a heterogeneous, unstable clinical profile, the challenge for the clinician is to detect the BPTs' persistence over time, interactions during the individual and family interviews, and what is reported by the adolescent about the relationships with his/her peers at school, on social networks, and, potentially, in hospital. The diagnostic challenge is to detect the "duration" criterion (1 year for $\left.D S M-V^{6}\right)$ and recurrent, abnormal functioning in several affective contexts. Another common presentation is nonsuicidal self-injury (NSSI). In recent studies, $58 \%$ of suicidal BPD adolescents reported NSSI, ${ }^{27}$ whereas $51.7 \%$ of female adolescents engaging in NSSI met criteria for BPD. ${ }^{43}$ Suicidal and NSSI behaviors should always prompt the clinician to screen for BPD. For the adolescent's physician, the syndrome might also manifest itself as repeated somatic problems ${ }^{42}$ or as poor adherence to the treatment of somatic complaint. ${ }^{44}$ Even though a growing number of clinicians recognize the clinical existence of BPD in the adolescent, there is still much debate as to the most appropriate way of conceptualizing this disorder (ie, in categorical or dimensional terms) as a single-factor or multifactor entity. ${ }^{30,45}$ In routine clinical practice, many clinicians adopt a categorical approach and refer to the diagnostic categories given in international classifications, including the $D S M-V .{ }^{6}$ However, several researchers have argued for an integrated developmental approach that takes account of both normal personality development and its anomalies. ${ }^{46}$ The clinician can therefore choose categorical or dimensional approaches for the diagnosis of BPD, together with a psychopathological approach for the identification of a BPO.

\section{The categorical approach}

In routine practice, the categorical approach refers to the $D S M-V$ diagnostic criteria ${ }^{6}$ (Table 1). The $D S M-V^{6}$ requires at least five of the criteria to be met. For a diagnosis of BPD, the traits must have been present for at least 1 year, and must be unchanging, inappropriate, and associated with a significant social or school-related impact or subjective suffering. Several validated instruments are available for this purpose: two clinician-rated interviews and three self-reporting tools are particularly popular. 


\section{Semi-structured clinical interviews}

These instruments should only be used by clinicians with appropriate training.

The DIB-R semi-structured interview is extensively used in research on BPD in adolescents. ${ }^{47}$ It includes 22 statements describing clinical dimensions pertaining to BPD. These statements are grouped into four sections: affect, cognition, impulsiveness, and interpersonal relationships. The DIB-R takes 45 minutes to administer, and the scoring requires clinical judgment. The score can be processed as a total score (ie, a quantitative index of BPD traits) or a cut-off score for diagnosing BPD. In the original publication, ${ }^{47}$ the interrater reliability ranged from 0.71 to 0.80 , and the testretest reliability of the total score $(\kappa=0.71)$ was good. Using a cut-off score of 8 , the DIB-R had a sensitivity of 0.82 and a specificity of 0.80 when compared with clinically derived psychiatric diagnoses.

The Childhood Interview for DSM-IV-TR Borderline Personality Disorder (CI-BPD) is a semi-structured interview that assesses BPD traits specifically in children and adolescents. ${ }^{48}$ It was derived from the borderline module of the Diagnostic Interview for Personality Disorders originally developed by Zanarini et al. The language was simplified, and certain forms of impulsivity were omitted. The CI-BPD's internal consistency and interrater reliability coefficients demonstrated adequate reliability. Concurrent validity was tested against the Child Behavior Checklist.

\section{Self-reports that can serve as a screening instrument}

1. The Abbreviated Diagnostic Interview for Borderlines (Ab-DIB) is a 26-item self-report screening instrument for BPD derived from the DIB-R. ${ }^{49}$ It encompasses the impulsiveness, affect, and cognitive components of the BPD construct and takes 10 minutes to administer. The Ab-DIB's psychometric properties were tested in suicidal adolescents. The internal consistencies and the test-retest intraclass correlation coefficients ranged from 0.80 to 0.86 and from 0.77 to 0.95 , respectively. Concurrent validity was tested against the DIB-R. A receiver operating characteristic analysis yielded an area under the curve (AUC) of 0.87 , indicating good diagnostic accuracy. The sensitivity was 0.88 , and the specificity ranged from 0.82 to 0.73 (depending on the age range).

2. The brief(11-item) Borderline Personality Features Scale for Children (BPFSC-11) is a shortened version of the original BPFSC, which in turn was initially derived from the Personality Assessment Inventory used with adults. ${ }^{50}$
It takes less than 10 minutes to administer. The BPFSC-11 has been evaluated for construct validity in community and clinical samples of adolescents. Concurrent validity was tested against the CI-BPD, yielding an AUC of 0.80 . The sensitivity was 0.74 , and the specificity was 0.71 .

3. The Dominic Interactive for Adolescents-Revised (DIA$\mathrm{R})$ is an interactive multimedia self-report screening for nine DSM-5 mental disorders (major depressive disorder [MDD], general anxiety disorder [AD], specific phobia, social phobia, obsessive compulsive disorder, attention deficit hyperactivity disorder [ADHD], oppositional defiant disorder [OD-CD], conduct disorder, and substance use disorder [SUD]), BPTs, and suicidality. ${ }^{51}$ It is available in French and English language versions, and takes 25 minutes to administer. The DIA-R's reliability and validity have been evaluated in community and clinical samples, demonstrating adequate test-retest reliability and internal consistency. Concurrent validity was tested against the Schedule for Affective Disorders and Schizophrenia for School-Aged Children. The AUCs ranged from high (0.91-0.94) to moderate (0.71-0.87), suggesting adequate accuracy in the prediction of mental disorders (other than OCD, for which the AUC was 0.61). When compared with the aforementioned self-reports, the DIA-R usefully screens for mental disorders as well as BPTs.

Differential diagnosis is challenging in adolescents. Subjects presenting essentially with NSSI behaviors may constitute a distinct group. The combination of self-injury behaviors with the cognitive symptoms of BPD (notably persecution ideation in stressful situations) is suggestive of the presence of BPD. The differential diagnosis vs bipolar disorder is particularly difficult, in as much as the two pathologies have similar sets of symptoms, marked by affective instability and self-injury behaviors. Very few mood-related criteria differentiate between the two disorders in the adolescent; only a frequent switch between a depressed mood and an elevated mood will indicate bipolar disorder. ${ }^{52}$ The lack of discriminant clinical elements will prompt the clinician to rely on additional investigations, such as actigraphy. When compared with depressed adolescents with bipolar disorder, nondepressed adolescents with BPD displayed a shorter, more variable total sleep time..$^{53}$

\section{The dimensional approach}

Although the discriminant, predictive validity of the categorical diagnosis of BPD has been established in the adolescent, several clinicians prefer to speak of personality traits rather 
than a disorder. ${ }^{54}$ This school of thought prompted the introduction of an alternative $D S M-V$ model for personality disorders, ${ }^{6}$ as a complement to the current categorical approach. The alternative BPD construct includes impairments in two or more of the following areas of personality functioning (identity, self-direction, empathy, and intimacy), and at least four pathological personality traits pertaining to negative affectivity, disinhibition, and antagonism. Identifying personality traits (rather than a disorder) is particularly appropriate when the clinician is uncertain of the symptoms' duration. Compared with the categorical approach, the dimensional diagnostic approach is more compatible with the many possible courses of the disorder. It therefore allows for a more open discussion of the prognosis with the adolescent and his/her parents.

\section{The psychopathological approach}

As a complement to the child psychiatry approach aimed at recording symptoms indicative of a diagnosis of BPD, a psychopathological evaluation can be used to explore the presence of a BPO with reference to the various domains of psychological functioning: the degree of identity integration, the level of defensive operations, and the capacity for reality testing. ${ }^{7}$ More generally, one situates the characteristic psychological functioning of BPO within the broad, diverse sets of psychological functioning and relationship patterns that coexist in the adolescent. The clinician identifies these aspects over the course of his/her interviews with the adolescent and the family, by analyzing the personal and family history of the adolescent, and, potentially, by relying on projective tests such as the Rorschach test, diagnostic psychodrama, and the "fairy tale of the ant" (a test exploring the youngster's body image). ${ }^{55}$

\section{Evaluation of the suicidal risk}

The high suicide rate associated with BPD should always prompt the clinician to explore the adolescent's suicidal ideation. In fact, $68 \%$ of the adolescents admitted to an emergency department after a suicide attempt presented with BPD. ${ }^{29}$ Active suicidal ideation is found in 59\% of adolescents with BPD, and NSSI is observed in 58\%. ${ }^{27}$ In addition, clinical studies consistently reported a higher frequency of suicidal attempts in BPD adolescents in comparison with non-BPD adolescents. ${ }^{24,27,40}$ The clinical evaluation of an adolescent with BPD should always involve an assessment of suicidal and NNSI behavior. Clinicians can rely on standardized instruments to investigate past and present suicidal symptoms, such as the Columbia Suicide Severity Rating
Scale. ${ }^{56}$ If the adolescent expresses suicidal ideation, the clinician has to evaluate the current suicidal risk by probing the following three domains: 1) risk factors and protective factors (family and peer adversity, stressful situations, social support, coping abilities, etc); 2) the degree of intent to act on suicidal thoughts and the plan (time, place, and method); and 3 ) access to a means of committing suicide, and the lethality of the planned method.

\section{Comorbidity}

All the clinical and epidemiological studies have emphasized the high frequency of comorbidities associated with BPD in the adolescent. ${ }^{2,57}$ The aforementioned epidemiological survey ${ }^{33}$ observed strong comorbidity with ADs and insomnia disorder, and very strong comorbidity with depressive disorders (DDs), ADHDs, and OD-CDs, with ORs ranging from 22 to 4 . When an adolescent is referred to an outpatient clinic for psychological difficulties, the parents rarely report insomnia. However, objective sleep disturbances are noted when this factor is specifically studied in adolescents with BPD. ${ }^{58}$ Clinical studies often confirm these comorbidities, leaving clinical researchers with the challenging task of disentangling depression and BPD. Studies of nondepressed adolescents with $\mathrm{BPD}^{27,53}$ and depression-adjusted multivariate analyses of mixed samples have confirmed that DD and BPD are distinct with regard to the diagnosis, suicidal risk, psychosocial correlates, and treatment efficacy. ${ }^{24}$ Clinical studies have highlighted two types of comorbidity: 1) true comorbidities, with the simultaneous but independent presence of a SUD or a $\mathrm{DD}^{59}$ and 2) integrated comorbidities, where BPD symptoms are intertwined with features of other $D S M-V$ disorders, such as disruptive mood dysregulation disorder, unspecified ADHD, unspecified feeding or eating disorder, unspecified dissociative disorder, and a newly developed non-DSM- $V$ diagnostic category referred to as disharmony. ${ }^{60}$

In clinical terms, comorbidities complicate the management of BPD in adolescence. They increase the suicidal risk (including the refusal to treat a chronic, severe, lifethreatening somatic disease $)^{44}$ and generally accentuate the disease burden. ${ }^{61}$

\section{The course of the disease, prevention, and prognosis}

Early adolescence is a period during which BPTs emerge within a complex clinical picture. The traits can either normalize or progress to BPD. This period is particularly delicate. Externalizing disorders in the preadolescent are 
associated with the occurrence of BPD in adolescence, as evidenced by both clinical studies ${ }^{62}$ and studies of the general population. ${ }^{42}$ Some epidemiological studies ${ }^{36}$ have shown that affective instability can be one of the first symptoms to emerge.

In the general population, BPTs are moderately stable just like other personality traits during adolescence, in fact. In contrast, the rank-order stability is strong, and so the most severely affected adolescents tend to remain symptomatic. ${ }^{63}$ This has been confirmed by clinical studies of suicidal cases of BPD; adolescents suffering from early onset, severe $\mathrm{BPD}$ are most at risk of seeing their disorder persist until adulthood. $^{28}$

From a developmental viewpoint, it is essential to consider comorbidities. In fact, the adolescent's outcome will differ according to whether the BPD is isolated or is associated with other disorders. ${ }^{46,59}$ A substantial degree of association has been observed when considering early onset mental disorders and personality psychopathology throughout adolescence and early adulthood. ${ }^{57}$ Longitudinal studies of clinical populations have investigated the relationship between BPD and ADHD, including the time course of their development. In a population of male adolescent outpatients, ADHD and oppositional defiant disorder (ODD) were found to be the only childhood disorders predictive of BPD symptoms in adolescence. ${ }^{62}$ The results of community studies support this observation. In a Canadian normative community cohort, ${ }^{42}$ childhood ADHD was predictive of BPD symptoms in adolescence. Likewise, in a US community study, ODD in 8- to 10 -year-old females and ADHD in 10- to 13-year-old females predicted the presence of BPD symptoms at the age of 14 years. ${ }^{64}$ These consistent findings emphasize: 1) the negative impact of childhood externalizing disorders (especially ODD) on subsequent personality development and 2) the need for more preventive interventions in childhood and more screening for BPTs in early adolescence.

Few studies have provided estimates of long-term stability. In a small sample of adolescent females with OPD, Biskin et $\mathrm{al}^{59}$ reported that the majority of youngsters having developed BPD in early adolescence remitted within 4 years. In contrast, Greenfield et $\mathrm{al}^{28}$ observed that $76 \%$ of suicidal adolescent females with BPD remained symptomatic 4 years later.

Several clinical studies have provided insight into the symptom-related prognosis for BPD and the transition to adulthood. According to these clinical studies, unstable relationships and an unstable self-image ${ }^{65}$ or affective instability ${ }^{59}$ with peaks of anger or sadness might be associated with the persistence of BPD into adulthood. The comorbidities and the family's contributions influence the progression of BPD. In studies of the general population, ${ }^{66}$ it has been found that the presence of BPD in the mother, an SUD in the father, mother-child conflicts, and depression or an SUD in the adolescent are associated with the persistence of BPD into adulthood. In clinical populations of adolescents, DDs and SUDs are associated with this persistence. ${ }^{59}$ The clinical studies have also shown that the early onset of BPD is linked to the persistence of the disorder into adulthood. ${ }^{28}$ It is noteworthy that in another study of an older community sample (14- to 18-year-old male and female adolescents), ${ }^{66}$ both externalizing and internalizing disorders in adolescence were associated with BPD symptoms in adulthood. However, multivariate analyses revealed that only the DDs and SUDs in adolescence predicted BPD in adulthood. Similar trajectories have been reported in clinical studies. ${ }^{59}$

Taken as whole, these data indicate that the presence of externalizing disorders in childhood predicts BPD symptoms at the beginning of adolescence, whereas depression in adolescence predicts BPD in adulthood. Both externalizing and internalizing disorders were comorbidities in BPD in midadolescence. ${ }^{25,28}$ Thus, certain researchers have advocated that BPD should rather be viewed as resulting from "a confluence of internalizing and externalizing problems". ${ }^{2}$ From a developmental perspective, one should be careful when children with ADHD or ODD switch to DD or display additional symptoms of DD in adolescence. Specific follow-up interventions should be provided to children and adolescents presenting with this developmental course.

\section{Therapeutic strategies}

Two general principles must be emphasized when considering the treatment of an adolescent with BPD. First, the treatment does not only correspond to the implementation of specific therapies but is also a management plan that relies on the therapists' ability to work cooperatively for the patient's benefit. Second, treatment requires commitment from the youngster and from the parents. The family definitively has a critical influence on treatment efficacy.

\section{Management}

Before initiating treatment, the therapist should obtain informed consent from both the youngster and his/her parent(s), in order to comply with the legislation and pave the way to a positive therapeutic alliance between the youngster, the parent(s), and the healthcare professionals ${ }^{67}$ As with any therapeutic intervention in adolescent medicine, parental 
involvement is critical. Considering the long duration of BPD and the unpredictability of its management, it is preferable to take the time to build a solid alliance with the youngster and his/her family, based on the provision of clear information about the pathology and its treatment, an evaluation of the adolescent's level of commitment, and the establishment of realistic treatment objectives. Family interviews are thus an essential component of any therapeutic intervention. Integrated care programs that offer an opportunity to review therapeutic transactions as a team and to re-evaluate the treatment options from time to time are more effective than a therapeutic intervention by an individual therapist. ${ }^{2}$ Regardless of the patient's status (ie, an outpatient or a partial or full inpatient), he/she must receive continuous, specialist care, with the management organized around outpatient consultations.

\section{Evidence-based pharmacotherapy}

It is important to determine the value of pharmacotherapy in the treatment plan. The Australian National Health and Medical Research Council ${ }^{68}$ and UK National Institute for Health and Care Excellence ${ }^{69}$ have reviewed the evidence from randomized controlled trials (RCTs). Both organizations recommended not prescribing psychotropic drugs in the treatment of BPD in adolescence. ${ }^{70}$ However, if the pharmacological approach must be used, it should be limited (according to the clinical trials evidence) to second generation antipsychotics. ${ }^{71}$ In the absence of high-quality evidence, selective serotonin reuptake inhibitors are not approved as treatments for BPD.

Second generation antipsychotics are useful adjuncts to psychological therapy in a suicide crisis $^{68}$ and can be prescribed as anxiolytics in the short time. Benzodiazepines should never be prescribed. In the long term, a nonpharmacological approach is preferable. The associated disorders and comorbidities may justify medication. Insomnia (frequently associated with BPD but often undiagnosed and untreated) should prompt the practitioner to consider the use of melatonin - either a standard formulation or prolonged release, depending on the type of insomnia. Some case series have evidenced an improvement. ${ }^{58}$ Moreover, methylphenidate and selective serotonin reuptake inhibitors are indicated for true comorbidities (ADHD and DD, respectively).

\section{Psychotherapy}

Supportive psychotherapy (SP) is the most commonly available therapy worldwide. Since the pioneering studies on cognitive behavioral therapy (CBT) in the early 2000s, much progress has been made on evaluating the efficacy of newly developed, specific psychotherapies. ${ }^{72}$ With regard to the therapeutic impact on BPTs and self-mutilating behavior, evidence-based data are available for several structured, manualized psychotherapies, including dialectic-behavioral therapy (DBT), cognitive analytic therapy (CAT), transference-focused therapy, and mentalization-based therapy $(\mathrm{MBT}){ }^{73}$

\section{Supportive psychotherapy}

Less impaired adolescents deserve SP, which is a usually available psychotherapy. It can be easily combined with case management and crisis intervention. Supportive psychotherapy thus meets the basic treatment needs of most adolescents with BPTs. Based on a nonjudgmental, empathizing approach, SP seeks to develop a shared problem formulation and increase the patient's levels of self-esteem and hope. ${ }^{74}$ The sessions can also include training in coping strategies. ${ }^{27,54}$ Supportive psychotherapy comprising weekly sessions with a case manager has been highlighted as a cost-effective means of addressing public health needs for BPD. ${ }^{75}$ In many countries, SP is most commonly used for BPD. Supportive psychotherapy has also been integrated into the diagnosis and early treatment of OPD in adolescents with BPTs. ${ }^{76}$ In fact, SP is the de facto first-line treatment for BPD. Off note, Gunderson has developed a structured management program which could be applied to BPD. The general ("good") psychiatric management (GPM) includes individual case management-orientated therapy focusing on situational stressors and social adaptation; medication, and family and group interventions are added as needed. ${ }^{8}$ No RCT has been conducted yet in BPD adolescents to the best of our knowledge.

\section{Dialectic behavioral therapy}

DBT has been adapted for adolescents. ${ }^{77,78}$ Dialectic-behavioral therapy for adolescents (DBT-A) consists of a three times weekly, multimodal therapeutic program that includes individual psychotherapy, multifamily skills training groups, family meetings, telephone coaching for patients and family members, and supervision for therapists. ${ }^{78}$ The relevance of between-session contacts should be evaluated in regular team meetings. ${ }^{79}$

Seventy-seven 15-year-old adolescents were enrolled in a 19-week RCT comparing DBT-A with treatment as usual (weekly psychodynamically oriented therapy or CBT) ${ }^{80}$ Both groups were receiving medication. The 1-year follow-up assessment demonstrated that DBT-A was associated with 
a greater long-term reduction in self-mutilating behaviors, suicidal ideation, and depressive and borderline symptoms. ${ }^{80}$

\section{Cognitive analytic therapy}

CAT incorporates components of psychodynamic psychotherapy and CBT.${ }^{70}$ Seventy-eight $15-18$-year-old outpatient adolescents with BPTs were randomly allocated to either CAT or manualized good clinical care. ${ }^{81}$ Both treatments were equally effective at the 24-month follow-up assessment, although CAT was associated with more rapid improvement of internalizing and externalizing symptoms. A newly designed RCT in 15-25-year-old youngsters with BPD is underway. ${ }^{82}$

\section{Mentalization-based therapy}

Mentalization refers to the capacity to understand (and thus predict) mental states and behaviors in oneself and others. ${ }^{18,83}$ It belongs to the social cognition processes that a child usually develops over time. In the context of attachment theory, mentalization refers to the notion of "reflective function", as defined by Fogy. ${ }^{18}$ The development of mentalization capacities relies on the quality of early parent-infant interactions. ${ }^{18}$ Adolescents with BPD displayed lower mentalization capacities than healthy controls. ${ }^{83}$ Two RCTs were recently conducted on MBT in adolescents (MBT-A). In the first study, ${ }^{84}$ eighty $12-17$-year-old adolescents ( $85 \%$ female) with BPD (as assessed with the CI-BPD - mentioned earlier), self-mutilating behaviors, and self-reported depression were randomly allocated to either MBT-A or "treatment as usual" (TAU). The 1-year therapeutic program included weekly individual sessions and monthly family MBT. The results showed that MBT-A was more effective than TAU in reducing BPD symptoms, self-mutilation, and depression. The positive effect of MBT-A on self-mutilating behavior was mediated by an improvement in mentalization and attachment abilities. A second RCT is now underway, with a group MBT-A format. ${ }^{85}$ The full 1-year MBT-A program encompassed an additional group MBT module. The preliminary results (although in the absence of a control group) revealed a favorable pre-/ posttreatment course, with improvements in borderline, depressive, and self-mutilation symptoms, and attachment and mentalization capacities. ${ }^{86}$

\section{Cognitive remediation and rehabilitation}

The metacognition impairments already documented in adults with $\mathrm{BPD}^{19}$ and the developmental delays in social cognition observed in adolescents with $\mathrm{BPD}^{54}$ argue in favor of cognitive remediation therapy. To the best of our knowledge, the putative efficacy of cognitive remediation in adolescents with BPD has not yet been studied. Even though adolescents with BPD do benefit from specific psychotherapy, a significant proportion of the individuals continue to experience functional impairment - thus justifying the use of cognitive rehabilitation. Promising results have been obtained in adults for both metacognitive ${ }^{87}$ and cognitive ${ }^{88}$ remediation.

\section{Treatment planning}

Drawing up a treatment plan with the adolescent and his/her parents and identifying drop-out factors are two essential components for therapeutic success. ${ }^{89}$ In order to avoid premature withdrawal from treatment, Desrosiers et a ${ }^{85}$ recommend that the therapist should clearly explain the rationale for treatment and clarify any false ideas concerning BPD, its outcomes, and its treatment. The nonpharmacological approach combines several themes into a clear, coherent treatment plan that is readily communicable to the adolescent and his/her parents:

1. Act on the suicide crisis. The prime objective is to ensure that the adolescent is safe (see previous section). Recurrence of suicidal ideation and behavior is prevented by acting not only on risk factors but also (and above all) on protective factors. ${ }^{90}$ After a suicide crisis, young people are followed up by phone and/or have a postemergency consultation. ${ }^{91}$ Adolescents who make suicide attempts should be given a safety plan, such as that developed by Stanley and Brown. ${ }^{92}$ Moreover, the therapist helps the adolescent to develop strategies for coping with stressors, ${ }^{27}$ his/her reasons to live, and his/her social support network. ${ }^{54}$

2. Act on the ongoing disorder and its impact. Supportive psychotherapy is the recommended first-line treatment, in as much as it is available at all psychiatric care services. According to the literature data, two forms of BPDspecific psychotherapy have been adapted for use with adolescents and tested in RCTs, with convincing efficacy results: $\mathrm{DBT}^{78}$ and $\mathrm{MBT}^{84}$ The RCT on CAT evidenced more rapid remission but not a differential effect on the symptoms themselves. ${ }^{77}$ Transference-focused psychotherapy (a form of psychotherapy inspired by psychoanalysis) has also been adapted for use with adolescents, with a view to the future performance of an RCT. ${ }^{93}$

3. Act on persistence factors, by treating comorbidities, addictions, and depression, and by changing the disabling environment (ie, the interactions with not only parents but also with siblings and peers). By collaborating with school health staff and telemedicine services, the therapist 
can 1) help the youngster to return to school; 2) support other stakeholders at the school and in the social services; and 3 ) provide a tailored school program.

4. Act preventively on family risk factors. Targeted primary prevention for children whose parents suffer from a personality disorder or an SUD should be initiated, in collaboration with the adult psychiatry services. In girls, frequent consultations during childhood for attention problems or oppositional deviant behavior should alert the clinician on the risk of developing personality disorders in adolescence, and so be monitored with particular attention. ${ }^{42}$ Secondary prevention by screening for BPD among adolescents who have gone through a suicide crisis can potentially be based on game-like, computerized self-questionnaires such as the Dominic Interactive for Adolescents (see the previous section). Tertiary prevention will include patient education, sleep hygiene, ${ }^{53}$ better stress management, training in coping strategies, ${ }^{27}$ the prevention of suicidal relapse, a safety plan, ${ }^{54,92}$ and cognitive rehabilitation.

In the last 10 or so years, innovative forms of psychotherapy have been assessed in RCTs. The psychotherapeutic armamentarium has therefore expanded considerably. ${ }^{72}$ However, the current literature relates to nonspecific components found in several types of recommended therapies: structured and clinicbased therapeutic interventions, training in suicide management, multiple treatment modalities, and weekly supervision meetings. ${ }^{8,94}$ In order to select treatment components, one must consider parameters such as 1) the psychotherapist's availability and willingness to treat; 2) the condition's severity, comorbidity, suicidality, and addiction components; and 3 ) the adolescent's level of motivation and parental support.

\section{Acknowledgments}

The review was achieved while the corresponding author was in residence as invited researcher at the Center for Research and Intervention on Suicide, Ethical Issues and End-of-life practices (CRISE), Montreal, Canada. The authors would like to thank Pr Mishara, Director, and the staff of CRISE for their support.

\section{Disclosure}

The authors report no conflicts of interest in this work.

\section{References}

1. Guilé JM, Greenfield B. Introduction personality disorders in childhood and adolescence. Can Child Adolesc Psychiatr Rev. 2004;13(3):51-52.

2. Sharp C, Fonagy P. Practitioner review: borderline personality disorder in adolescence - recent conceptualization, intervention, and implications for clinical practice. J Child Psychol Psychiatry. 2015;56(12):1266-1288.
3. Kaess M, Brunner R, Chanen A. Borderline personality disorder in adolescence. Pediatrics. 2014;134(4):782-793.

4. Speranza M, Pham-Scottez A, Revah-Levy A, et al. Factor structure of borderline personality disorder symptomatology in adolescents. Can J Psychiatry. 2012;57(4):230-237.

5. Becker DF, Mcglashan TH, Grilo CM. Exploratory factor analysis of borderline personality disorder criteria in hospitalized adolescents. Compr Psychiatry. 2006;47(2):99-105.

6. American Psychiatric Association. Diagnostic and Statistical Manual of Mental Disorders. 5th ed. Arlington, VA: American Psychiatric Association; 2013.

7. Kernberg OF, Goldstein EG, Carr AC, Hunt HF, Bauer SF, Blumenthal R. Diagnosing borderline personality. A pilot study using multiple diagnostic methods. J Nerv Ment Dis. 1981;169(4):225-231.

8. Gunderson JG, Herpertz SC, Skodol AE, Torgersen S, Zanarini MC. Borderline personality disorder. Nat Rev Dis Primers. 2018;4:18029.

9. Plener PL, Bubalo N, Fladung AK, Ludolph AG, Lulé D. Prone to excitement: adolescent females with non-suicidal self-injury (NSSI) show altered cortical pattern to emotional and NSS-related material. Psychiatry Res. 2012;203(2-3):146-152.

10. Leboeuf A, Guilé JM, Labelle R, Luck D. Functional neuroimaging pilot study of borderline personality disorder in adolescents. Sante Ment Que;41(1):141-162.

11. Bourvis N, Aouidad A, Cabelguen C, Cohen D, Xavier J. How do stress exposure and stress regulation relate to borderline personality disorder? Front Psychol. 2017;8:2054.

12. Kaufman J. Reply to: citation distortions in the literature on the serotonin-transporter-linked polymorphic region and Amygdala activation. Biol Psychiatry. 2015;78(8):e37-e38.

13. Cicchetti D, Handley ED. Methylation of the glucocorticoid receptor gene, nuclear receptor subfamily 3 , group $\mathrm{C}$, member 1 (NR3C1), in maltreated and nonmaltreated children: associations with behavioral undercontrol, emotional lability/negativity, and externalizing and internalizing symptoms. Dev Psychopathol. 2017;29(5):1795-1806.

14. Stepp SD, Whalen DJ, Scott LN, Zalewski M, Loeber R, Hipwell AE. Reciprocal effects of parenting and borderline personality disorder symptoms in adolescent girls. Dev Psychopathol. 2014;26(2):361-378.

15. Steele M, Bate J, Nikitiades A, Buhl-Nielsen B. Attachment in adolescence and borderline personality disorder. J Infant Child Adolesc Psychother. 2015;14(1):16-32.

16. Steele H, Siever L. An attachment perspective on borderline personality disorder: advances in gene-environment considerations. Curr Psychiatry Rep. 2010;12(1):61-67.

17. Bick E. The experience of the skin in early object-relations. Int J Psychoanal. 1968;49(2):484-486.

18. Fonagy P, Bateman A. The development of borderline personality disorder - a mentalizing model. J Pers Disord. 2008;22(1):4-21.

19. Semerari A, Carcione A, Dimaggio G, Nicoló G, Pedone R, Procacci M. Metarepresentative functions in borderline personality disorder. $J$ Pers Disord. 2005;19(6):690-710.

20. Orts Clemmensen LM. Olrik Wallenstein Jensen S, Zanarini MC, Skadhede S, Munk-Jorgensen P. Changes in treated incidence of borderline personality disorder in Denmark: 1970-2009. Can J Psychiatry. 2013;58(9):522-528.

21. Cailhol L, Pelletier É, Rochette L, et al. Prevalence, mortality, and health care use among patients with cluster B personality disorders clinically diagnosed in Quebec: a provincial cohort study, 2001-2012. Can J Psychiatry. 2017;62(5):336-342.

22. Olajide K, Tyrer P, Singh SP, et al. Likelihood and predictors of detention in patients with personality disorder compared with other mental disorders: a retrospective, quantitative study of Mental Health Act assessments. Personal Ment Health. 2016;10(3):191-204.

23. Chanen AM, Jackson HJ, Mcgorry PD, Allot KA, Clarkson V, Yuen HP. Two-year stability of personality disorder in older adolescent outpatients. J Pers Disord. 2004;18(6):526-541. 
24. Sharp C, Green KL, Yaroslavsky I, Venta A, Zanarini MC, Pettit J. The incremental validity of borderline personality disorder relative to major depressive disorder for suicidal ideation and deliberate self-harm in adolescents. J Pers Disord. 2012;26(6):927-938.

25. Ha C, Balderas JC, Zanarini MC, Oldham J, Sharp C. Psychiatric comorbidity in hospitalized adolescents with borderline personality disorder. J Clin Psychiatry. 2014;75(5):e457-e464.

26. Becker DF, Grilo CM, Edell WS, Mcglashan TH. Diagnostic efficiency of borderline personality disorder criteria in hospitalized adolescents: comparison with hospitalized adults. Am J Psychiatry. 2002;159(12):2042-2047.

27. Knafo A, Guilé JM, Breton JJ, et al. Coping strategies associated with suicidal behaviour in adolescent inpatients with borderline personality disorder. Can J Psychiatry. 2015;60(2 Suppl 1):S46-S54.

28. Greenfield B, Henry M, Lis E, et al. Correlates, stability and predictors of borderline personality disorder among previously suicidal youth. Eur Child Adolesc Psychiatry. 2015;24(4):397-406.

29. Greenfield B, Henry M, Weiss M, et al. Previously suicidal adolescents: predictors of six-month outcome. J Can Acad Child Adolesc Psychiatry. 2008;17(4):197-201.

30. Tyrer P, Reed GM, Crawford MJ. Classification, assessment, prevalence, and effect of personality disorder. Lancet. 2015;385(9969): 717-726.

31. Bernstein DP, Cohen P, Velez CN, Schwab-Stone M, Siever LJ, Shinsato L. Prevalence and stability of the DSM-III-R personality disorders in a community-based survey of adolescents. Am J Psychiatry. 1993;150(8):1237-1243.

32. Johnson JG, Cohen P, Kasen S, Skodol AE, Oldham JM. Cumulative prevalence of personality disorders between adolescence and adulthood Acta Psychiatr Scand. 2008;118(5):410-413.

33. Guile JM, Berthiaume C, Bergeron L. Prevalence and comorbidity of borderline personality traits in the Quebec general population 12 to 14 years of age. In: 53rd Annual Meeting; October 24-29, 2006; San Diego: American Academy of Child and Adolescent Psychiatry.

34. Bird HR, Shaffer D, Fisher P, Gould M, Stachezza B, Chen JY. The Columbia Impairment Scale (CIS): pilot findings on a measure of global impairment for children and adolescents. Int J Psychiatric Res. 1993;3:167-176.

35. Chabrol H, Montovany A, Chouicha K, Callahan S, Mullet E. Frequency of borderline personality disorder in a sample of French high school students. Can J Psychiatry. 2001;46(9):847-849.

36. Zanarini MC, Horwood J, Wolke D, Waylen A, Fitzmaurice G, Grant BF. Prevalence of DSM-IV borderline personality disorder in two community samples: 6,330 English 11-year-olds and 34,653 American adults. J Pers Disord. 2011;25(5):607-619.

37. Leung SW, Leung F. Construct validity and prevalence rate of borderline personality disorder among Chinese adolescents. J Pers Disord. 2009;23(5):494-513.

38. Moran P, Crawford MJ. Assessing the severity of borderline personality disorder. Br J Psychiatry. 2013;203(3):163-164.

39. Breton JJ, Bergeron L, Valla JP, et al. Quebec child mental health survey: prevalence of DSM-III-R mental health disorders. J Child Psychol Psychiatry. 1999;40(3):375-384.

40. Yen S, Gagnon K, Spirito A. Borderline personality disorder in suicidal adolescents. Personal Ment Health. 2013;7(2):89-101.

41. Fonseca-Pedrero E, Paino M, Lemos-Giráldez S, Muñiz J. Cluster B maladaptive personality traits in Spanish adolescents. Rev Psiquiatr Salud Ment. 2013;6(3):129-138.

42. Haltigan JD, Vaillancourt T. Identifying trajectories of borderline personality features in adolescence: antecedent and interactive risk factors. Can J Psychiatry. 2016;61(3):166-175.

43. Nock MK, Joiner TE, Gordon KH, Lloyd-Richardson E, Prinstein MJ. Non-suicidal self-injury among adolescents: diagnostic correlates and relation to suicide attempts. Psychiatry Res. 2006;144(1):65-72.

44. Jaunay E, Consoli A, Greenfield B, Guilé JM, Mazet P, Cohen D. Treatment refusal in adolescents with severe chronic illness and borderline personality disorder. $J$ Can Acad Child Adolesc Psychiatry. 2006;15(3):135-142.
45. Sharp C. Calling for a unified redefinition of "Borderlineness": commentary on Gunderson et al. J Pers Disord. 2018;32(2):168-174.

46. Cicchetti D. Illustrative developmental psychopathology perspectives on precursors and pathways to personality disorder: commentary on the special issue. J Pers Disord. 2014;28(1):172-179.

47. Zanarini MC, Gunderson JG, Frankenburg FR, Chauncey DL. The Revised Diagnostic Interview for Borderlines: Discriminating BPD from other Axis II Disorders. J Pers Disord. 1989;3(1):10-18.

48. Sharp C, Ha C, Michonski J, Venta A, Carbone C. Borderline personality disorder in adolescents: evidence in support of the Childhood Interview for DSM-IV borderline personality disorder in a sample of adolescent inpatients. Compr Psychiatry. 2012;53(6):765-774.

49. Guilé JM, Greenfield B, Berthiaume C, Chapdelaine C, Bergeron L. Reliability and diagnostic efficiency of the abbreviated-diagnostic interview for borderlines in an adolescent clinical population. Eur Child Adolesc Psychiatry. 2009;18(9):575-581.

50. Sharp C, Steinberg L, Temple J, Newlin E. An 11-item measure to assess borderline traits in adolescents: refinement of the BPFSC using IRT. Personal Disord. 2014;5(1):70-78.

51. Bergeron L, Smolla N, Berthiaume C, et al. Reliability, validity, and clinical utility of the dominic interactive for adolescents-revised A DSM-5-based self-report screen for mental disorders, borderline personality traits, and suicidality. Can J Psychiatry. 2017;62(3):211-22.

52. Huynh C. Rythme veille-sommeil et dimensions cliniques dans le trouble de personnalité limite à l'adolescence (Sciences biomédicales, option sciences psychiatriques). Montreal: Université de Montréal; 2013. French.

53. Huỳnh C, Guilé JM, Breton JJ, Godbout R. Sleep-wake patterns of adolescents with borderline personality disorder and bipolar disorder. Child Psychiatry Hum Dev. 2016;47(2):202-214.

54. Garny de La Rivière S, Knafo A, Pripis C, Rey N, Guilé JM. [Borderline personality disorder in adolescence]. Les troubles de la personnalité limite l'adolescence Lett Psychiatre. 2014;10(3):92-97. French.

55. de Tychey C. Comparative genetic approach to the study of body image of children from 4 to 14 years of age using the fairy tale of the ant. Percept Mot Skills. 1993;76(3 Pt 2):1179-1189.

56. Posner K, Brown GK, Stanley B, et al. The Columbia-Suicide Severity Rating Scale: initial validity and internal consistency findings from three multisite studies with adolescents and adults. Am J Psychiatry. 2011;168(12):1266-1277.

57. Lewinsohn PM, Rohde P, Seeley JR, Klein DN. Axis II psychopathology as a function of Axis I disorders in childhood and adolescence. $J \mathrm{Am}$ Acad Child Adolesc Psychiatry. 1997;36(12):1752-1759.

58. Guilé JM, Huynh C, Desrosiers L, et al. Exploring sleep disturbances in adolescent borderline personality disorder using actigraphy: a case report. Int J Adolesc Med Health. 2009;21(1):123-126.

59. Biskin RS, Paris J, Renaud J, Raz A, Zelkowitz P. Outcomes in women diagnosed with borderline personality disorder in adolescence. $J$ Can Acad Child Adolesc Psychiatry. 2011;20(3):168-174.

60. Xavier J, Vannetzel L, Viaux S, et al. Reliability and diagnostic efficiency of the Diagnostic Inventory for Disharmony (DID) in youths with pervasive developmental disorder and multiple complex developmental disorder. Res Autism Spectrum Disorders. 2011;5(4):1493-1499.

61. Feenstra DJ, Hutsebaut J, Laurenssen EM, Verheul R, Busschbach JJ, Soeteman DI. The burden of disease among adolescents with personality pathology: quality of life and costs. J Pers Disord. 2012;26(4): 593-604.

62. Burke JD, Stepp SD. Adolescent disruptive behavior and borderline personality disorder symptoms in young adult men. J Abnorm Child Psychol. 2012;40(1):35-44.

63. Bornovalova MA, Hicks BM, Iacono WG, Mcgue M. Longitudinal twin study of borderline personality disorder traits and substance use in adolescence: developmental change, reciprocal effects, and genetic and environmental influences. Personal Disord. 2013;4(1):23-32.

64. Stepp SD, Burke JD, Hipwell AE, Loeber R. Trajectories of attention deficit hyperactivity disorder and oppositional defiant disorder symptoms as precursors of borderline personality disorder symptoms in adolescent girls. J Abnorm Child Psychol. 2012;40(1):7-20. 
65. Garnet KE, Levy KN, Mattanah JJ, Edell WS, Mcglashan TH. Borderline personality disorder in adolescents: ubiquitous or specific? Am J Psychiatry. 1994;151(9):1380-1382.

66. Stepp SD, Olino TM, Klein DN, Seeley JR, Lewinsohn PM. Unique influences of adolescent antecedents on adult borderline personality disorder features. Personal Disord. 2013;4(3):223-229.

67. National Collaborating Centre for Mental Health. Borderline Personality Disorder: Treatment and Management. Leicester: NICE (National Institute for Health and Care Excellence); 2009.

68. Chanen AM, Thompson KN. Prescribing and borderline personality disorder. Aust Prescr. 2016;39(2):49-53.

69. National Collaborating Centre for Mental Health. Borderline Personality Disorder: The NICE Guideline on Treatment and Management. National Clinical Practice Guideline No 78. National Institute for Health and Clinical Excellence. Leicester, UK: The British Psychological Society \& The Royal College of Psychiatrists; 2009.

70. Biskin RS. Treatment of borderline personality disorder in youth. $J$ Can Acad Child Adolesc Psychiatry. 2013;22(3):230-234.

71. Stoffers JM, Lieb K. Pharmacotherapy for borderline personality disorder - current evidence and recent trends. Curr Psychiatry Rep. 2015;17(1):534.

72. Guilé JM, Greenfield B, Breton JJ, Cohen D, Labelle R. Is psychotherapy effective for borderline adolescents? Clin Neuropsychiatr. 2005;2(5):277-282.

73. Ougrin D, Latif S. Specific psychological treatment versus treatment as usual in adolescents with self-harm: systematic review and metaanalysis. Crisis. 2011;32(2):74-80.

74. Philip NS, Rost-Banik D, Shaffer S, Johnson B, Boland R. Supportive psychotherapy: a crash course for medical students. Acad Psychiatry. 2010;34(1):57-60.

75. Gunderson JG. The emergence of a generalist model to meet public health needs for patients with borderline personality disorder. $\mathrm{Am} \mathrm{J}$ Psychiatry. 2016;173(5):452-458.

76. Chanen AM, Mccutcheon L. Prevention and early intervention for borderline personality disorder: current status and recent evidence. $\mathrm{Br}$ J Psychiatry Suppl. 2013;54:s24-s29.

77. Garny de La Rivière S, Houinou Ebo BBN, Segard V, et al. Adapting dialectical behavioral therapy to francophone adolescents, a pilot study in female adolescents with major depressive disorder and borderline personality disorder. Neuropsychiatr Enfance Ado. 2017;65:5-13.

78. Klein DA, Miller AL. Dialectical behavior therapy for suicidal adolescents with borderline personality disorder. Child Adolesc Psychiatr Clin NAm. 2011;20(2):205-216.

79. Jacob KL. Clinical observations about the potential benefits and pitfalls of between-session contacts with borderline patients. Harv Rev Psychiatry. 2016;24(5):e8-e14.

80. Mehlum L, Ramberg M, Tørmoen AJ, et al. Dialectical behavior therapy compared with enhanced usual care for adolescents with repeated suicidal and self-harming behavior: outcomes over a one-year follow-up. J Am Acad Child Adolesc Psychiatry. 2016;55(4):295-300.
81. Chanen AM, Jackson HJ, Mccutcheon LK, et al. Early intervention for adolescents with borderline personality disorder: quasi-experimental comparison with treatment as usual. Aust $N Z J$ Psychiatry. 2009;43(5):397-408.

82. Chanen A, Jackson H, Cotton SM, et al. Comparing three forms of early intervention for youth with borderline personality disorder (the MOBY study): study protocol for a randomised controlled trial. Trials. 2015; 16:476.

83. Quek J, Melvin GA, Bennett C, Gordon MS, Saeedi N, Newman LK. Mentalization in adolescents with borderline personality disorder: a comparison with healthy controls. J Pers Disord. 2018:1-19. Epub $2018 \mathrm{Feb} 22$.

84. Rossouw TI, Fonagy P. Mentalization-based treatment for self-harm in adolescents: a randomized controlled trial. J Am Acad Child Adolesc Psychiatry. 2012;51(12):1304-1313.

85. Beck E, Bo S, Gondan M, et al. Mentalization-based treatment in groups for adolescents with borderline personality disorder (BPD) or subthreshold BPD versus treatment as usual (M-GAB): study protocol for a randomized controlled trial. Trials. 2016;17(1):314.

86. Bo S, Sharp C, Beck E, Pedersen J, Gondan M, Simonsen E. First empirical evaluation of outcomes for mentalization-based group therapy for adolescents with BPD. Personal Disord. 2017;8(4): 396-401.

87. Schilling L, Moritz S, Kriston L, Krieger M, Nagel M. Efficacy of metacognitive training for patients with borderline personality disorder: preliminary results. Psychiatry Res. 2018;262:459-464.

88. Pascual JC, Palomares N, Ibáñez Á, et al. Efficacy of cognitive rehabilitation on psychosocial functioning in borderline personality disorder: a randomized controlled trial. BMC Psychiatry. 2015;15:255.

89. Desrosiers L, Saint-Jean M, Breton JJ. Treatment planning: a key milestone to prevent treatment dropout in adolescents with borderline personality disorder. Psychol Psychother. 2015;88(2):178-196.

90. Breton JJ, Labelle R, Berthiaume C, et al. Protective factors against depression and suicidal behaviour in adolescence. Can J Psychiatry. 2015;60(2 Suppl 1):S5-S15.

91. Mirkovic B, Belloncle V, Rousseau C, Knafo A, Guilé J-M, Gérardin P. Stratégies de prévention du suicide et des conduites suicidaires l'adolescence : revue systématique de la littérature [Prevention strategies of suicide and suicidal behavior in adolescents; a systematic review]. Neuropsychiatrie de l'enfance et de l'adolescence. 2014;6246:33. French.

92. Stanley B, Brown GK. Safety planning intervention: a brief intervention to mitigate suicide risk. Cogn Behav Practice. 2012;19(2):256-264.

93. Foelsch PA, Odom AE, Kernberg OF. Treatment of adolescents with identity diffusion: a modification of transference focused psychotherapy. Sante Ment Que. 2008;33(1):37-60.

94. Ougrin D, Tranah T, Stahl D, Moran P, Asarnow JR. Therapeutic interventions for suicide attempts and self-harm in adolescents: systematic review and meta-analysis. JAm Acad Child Adolesc Psychiatry. 2015;54(2):97-107.
Adolescent Health, Medicine and Therapeutics

Publish your work in this journal

Adolescent Health, Medicine and Therapeutics is an international, peer-reviewed, open access journal focusing on health, pathology, and treatment issues specific to the adolescent age group. All aspects of health maintenance, preventative measures and disease treatment interventions are addressed within the journal and practitioners from all disciplines are
Dovepress

invited to submit their work as well as healthcare researchers and patient support groups. This journal is included in PubMed. The manuscript management system is completely online and includes a very quick and fair peer-review system. Visit http://www.dovepress.com/testimonials. php to read real quotes from published authors. 